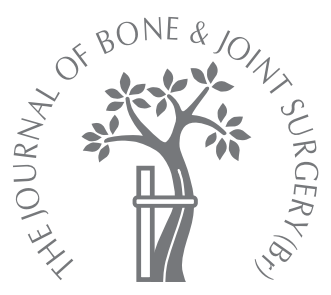

A. M. Foruria, J. W. Sperling, H. K. Ankem, L. S. Oh, R. H. Cofield

From the Mayo Clinic, Rochester, Minnesota, United States

A. M. Foruria, $\mathrm{MD}, \mathrm{PhD}$, Orthopaedic Surgeon Shoulder and Elbow Reconstructive Surgery Unit Fundacion Jimenez Diaz-Capio Avda Reyes Catolicos 2, 28040 Madrid, Spain.

J. W. Sperling, MD, MBA Professor of Orthopaedics R. H. Cofield, MD, Emeritus Professor of Orthopaedics Department of Orthopaedic Surgery

Mayo Clinic, 200 First Stree Southwest, Rochester Minnesota 55905, USA

H. K. Ankem, MD Orthopaedic Surgeon L. S. Oh, MD, Instructor in Orthopaedic Surgery Harvard University 175 Cambridge Street, Su 400 Boston, Massachusetts 02114, USA.

Correspondence should be sent to Professor R. H. Cofield; email: cofield.robert@mayo.edu

(c)2010 British Editorial Society of Bone and Joint Surgery doi:10.1302/0301-620X.92B7 $23671 \$ 2.00$

$J$ Bone Joint Surg [Br] 2010;92-B:970-4.

Received 12 October 2009;

Accepted after revision 16

March 2010

\title{
Total shoulder replacement for osteoarthritis in patients 80 years of age and older
}

This study was performed to review the safety and outcome of total shoulder replacements in patients who are $\geq \mathbf{8 0}$ years of age. A total of $\mathbf{5 0}$ total shoulder replacements in 44 patients at a mean age of 82 years ( 80 to 89 ) were studied. Their health and shoulder status, the operation and post-operative course were analysed, including pain, movement, patient satisfaction, medical and surgical complications, radiographs, the need for revision surgery, and implant and patient survival. A total of 27 patients had an ASA classification of III or IV and medical abnormalities were common. Of the 13 shoulders with bony deficiency of the glenoid, nine required grafting. The duration of hospital stay was prolonged and blood transfusions were common. There were no peri-operative deaths. The mean follow-up was for 5.5 years ( 2 to 12 ). Pain was significantly reduced $(p<0.001)$ and movement improved in active elevation and both external and internal rotation $(p<0.001)$. Using the Neer scale for assessing outcome, $40(80 \%)$ shoulders had an excellent or satisfactory result. There were medical or surgical complications in 17 cases. Four shoulders developed radiological evidence of loosened glenoid components, and three of these had a poor outcome. Three other shoulders required revision, two for instability. By the time of this review 39 of the patients had died from unrelated causes at a mean of 7.5 years (0.8 to 16.4) after surgery.

Total shoulder replacement is a relatively effective treatment in this elderly group of patients. However, there is a requirement for more intense patient care in the peri-operative period, and non-fatal medical or surgical complications are common. Most of these elderly patients will have a comfortable functional shoulder for the rest of their lives.

Primary osteoarthritis (OA) is the most common indication for total shoulder replacement (TSR). ${ }^{1}$ Little information exists about the safety and efficacy of shoulder replacement in the elderly ${ }^{2,3}$ compared to other age groups $^{4,5}$ and the general population. ${ }^{6-8}$ There are several factors which the surgeon may take into account before recommending TSR for the elderly patient with severe OA including the impact of surgery on the patient's general health, the possibility of significant glenoid wear and osteopenia, the stronger consideration for fixation of the humeral component with cement due to poor bone quality, ${ }^{9}$ lesser functional gains, the development of instability due to compromised quality of the soft-tissue, limited data on the survival of the implants, and the possibility of the need for a revision operation at an even older age. The aim of this study was to review the safety, efficacy and complication rate of primary TSR in patients with primary OA who are $\geq 80$ years of age.

\section{Materials and Methods}

The study was conducted at the Mayo Clinic following institutional approval. Our joint replacement registry identified 1473 primary TSRs (48\% in men) of an anatomic design performed between 1980 and 2000: 85 were performed in patients $\geq 80$ years of age, 51 of whom had primary OA, and on the dominant side in 26 patients. Only four hemiarthroplasties were implanted during this period for patients with OA in this age group. The 51 consecutive TSRs performed in 45 patients with primary OA form the basis of this study. One patient died within the first year after operation from unrelated causes. The remaining 44 patients ( 50 shoulders) were followed for a mean of 5.5 years (2 to 12 ). Preand immediate post-operative radiographs were available for review. Pre-operative radiographs were analysed for glenoid deficiency, classified by location and severity. ${ }^{10}$ The final radiographs were available at a mean of three years (immediately post-operative to 7.2 
Table I. Pre-operative patient comorbidities

\begin{tabular}{lll}
\hline & Patients & Percentage \\
\hline High blood pressure & 25 & 50 \\
Myocardial infarction & 13 & 26 \\
Atrial fibrillation & 8 & 16 \\
Hypothyroidism & 6 & 12 \\
Deep-vein thrombosis & 6 & 12 \\
Asthma/chronic obstructive pulmonary disease & 5 & 10 \\
Chronic anaemia & 5 & 10 \\
Cerebrovascular accident & 3 & 6 \\
Diabetes mellitus & 3 & 6 \\
Renal insufficiency & 3 & 6 \\
Morbid obesity & 3 & 6 \\
\hline
\end{tabular}

years). In two shoulders only the immediate post-operative studies were available. In eight shoulders the last radiograph available was obtained during the first two years of follow-up, and in 40 shoulders two or more years of radiological follow-up was possible. Lucent lines were assessed according to width and location. ${ }^{11}$

The mean age of the patients at the time of arthroplasty was 82 years ( 80 to 89 ). There were 34 arthroplasties performed in women and 16 in men. In 16 there were no medical comorbidities, but 18 had three or more. A list of preoperative comorbidities can be found in Table I. The mean body mass index was 27 (20 to 42). The American Society of Anaesthesiologists (ASA) classification was level I in one shoulder, level II in 22 , level III in 26 , and level IV in one. ${ }^{12}$

A total of 34 arthroplasties were performed under general anaesthesia plus regional anaesthesia using an interscalene block. In 15 , only a general anaesthetic was used, and in one, only regional anaesthesia. There were 45 procedures carried out through a deltopectoral approach and five through an anteromedial approach, detaching the anterior deltoid from the clavicle and anterior acromion. Before 1992 anteromedial approaches had been used. ${ }^{13}$ There was a high-grade partial-thickness rotator cuff tear in one shoulder. Four shoulders had full-thickness rotator cuff tears. They were measured intra-operatively, being small in two $(\leq 1 \mathrm{~cm}$ in largest diameter) and medium-sized in two ( $>1 \mathrm{~cm}$ to $3 \mathrm{~cm}$ in diameter). The subscapularis was incised through its tendon in 39 shoulders and was detached from bone in 11, in order to improve external rotation at the time of reattachment. Pre-operative radiological analysis and intra-operative findings identified 13 shoulders with moderate to severe deficiency of the glenoid bone. This was located posteriorly in eight, anteriorly in three, inferiorly in one and centrally in one. Autograft was added from the resected humeral head in nine shoulders, six using corticocancellous and three cancellous bone, with the remaining deficiencies managed by glenoid reaming. All patients received a Cofield implant (Smith and Nephew, Memphis, Tennessee). All-polyethylene glenoid components were used in 30 shoulders and metal-backed components in 20. Glenoid fixation included the use of cement in 41 arthro- plasties and uncemented bone ingrowth in nine. The humeral component was press-fitted in 42 arthroplasties. Cement was used for fixation in eight when there was poor bone quality proximally or the stem-bone fit distally was not firm. A drain was used post-operatively in 34 cases.

The hospital course was carefully evaluated, including the duration of stay, the development of medical complications or wound problems, and the need for blood transfusion or stay in the intensive-care unit. Pain was evaluated pre- and post-operatively as $1=$ no pain, $2=$ slight, $3=$ moderate after unusually heavy activity, $4=$ moderate and $5=$ severe. Patient satisfaction was rated as $1=$ much better, 2 = better, $3=$ the same, and $4=$ worse compared to before operation. Pre- and post-operative movement was measured with a goniometer by a surgeon as part of the patient's evaluation, and recorded on specific sheets. Active elevation and external rotation were measured in degrees, and for internal rotation the most proximal spinal level reached by the thumb was recorded. As the study began in 1980 , outcome was assessed using the modified Neer rating system. ${ }^{4}$ The result was excellent when the patient had no or slight pain, active abduction to at least $140^{\circ}$, external rotation to at least $45^{\circ}$, and was satisfied. The result was satisfactory when the patient had no or slight pain or moderate pain only with vigorous activity, active abduction to at least $90^{\circ}$, external rotation to at least $20^{\circ}$, and was satisfied. If any of the criteria were not met, the result was unsatisfactory. Patients who had an additional operative procedure also had an unsatisfactory result. Delayed clinical and radiological complications were assessed, as was the need for revision and survival of the implant.

Statistical analysis. Data were analysed with SPSS 17.0 Software (SPSS Inc., Chicago, Illinois). Continuous variables were contrasted with a normal distribution by the Kolmogorov-Smirnov test. Movement in elevation and external rotation before and after surgery was compared with paired $t$-tests. Pain and movement in internal rotation before and after surgery were compared with Wilcoxon's signed-ranks test. Comparisons for pain, movement and duration of stay between patient groups were performed with the Mann-Whitney test. Association between glenoid erosion and the rate of glenoid loosening, and graft use and glenoid loosening, were tested with chi-squared and Fisher's exact tests.

\section{Results}

The mean hospital stay was seven days (2 to 22). Before 1995 (26 shoulders) the mean stay was 9.5 days (3 to 22 ), and after 1995 (25 shoulders) 4.4 days (3 to 11); which was statistically significant $(\mathrm{p}<0.001)$. Admission to the intensive-care unit was required for five patients, three for 24 hours only. The mean reduction in haemoglobin was $3.2 \mathrm{~g} / \mathrm{dl}$ (2 to 7). Blood transfusions were used after 19 shoulder replacements, with one unit being given in four, two units in 12 and three units in three. In 41 cases there were no medical complications in the post-operative 
period. Four patients had cardiac complications, three with signs of cardiac insufficiency or a change in cardiac rhythm, and one with post-operative infarction. There was severe electrolyte imbalance, urinary retention, deepvein thrombosis, a wound haematoma and paraesthesia in the axillary nerve distribution in one case each. There were no peri-operative deaths.

Overall, the mean pain rating improved from 4.9 to 1.8 $(\mathrm{p}<0.001)$. Post-operative pain was rated as none in 29 shoulders, slight in ten, moderate after unusually heavy activity in four, moderate in four, and severe in three. Mean elevation improved from $80^{\circ}$ to $138^{\circ}$ ( $\mathrm{p}<0.001,95 \%$ CI 41 to 75$)$, mean external rotation from $19^{\circ}$ to $48^{\circ}$ (p < 0.001, CI $95 \% 18$ to 41$)$ and mean internal rotation from the sacrum to $\mathrm{L} 2(\mathrm{p}<0.001)$. The patients' assessment of their surgery was much better in 28 , better in 11 , the same in seven and worse in four. The Neer result rating showed the outcome to be excellent in 30 shoulders, satisfactory in ten and unsatisfactory in ten. The reason for an unsatisfactory result was moderate or severe pain in four, moderate or severe pain and lack of movement (elevation $<90^{\circ}$ and/or external rotation < $20^{\circ}$ ) in three, and lack of movement only in three.

Post-operatively, five shoulders developed anterosuperior subluxation of the humeral head. One shoulder continued to have excellent pain relief and mobility and no further treatment was required. One with a pre-existing medium-sized tear of the rotator cuff also had no significant pain and enough function to perform basic activities of daily living. The third shoulder with this complication had moderate pain with unusually heavy activities, active elevation of $60^{\circ}$ and external rotation of $0^{\circ}$, but declined further surgery. Two of the five patients in this group underwent further surgery. One had moderate to severe pain and pseudoparalysis associated with the anterosuperior subluxation. This required revision of the components and repair of the rotator cuff. Unfortunately, the patient did not improve and a further revision was performed. The other revision procedure was for anterosuperior subluxation included repair of the rotator cuff and exchange of the glenoid component for substantial wear. The surgery reduced the patients pain and marginally improved movement and function.

The early post-operative radiographs were satisfactory in all but one patient, in whom the tip of the stem had perforated the cortex of the humerus. Early revision was performed, but unfortunately the patient did not do well, with continuing severe pain and impaired movement because of posterior dislocation of the prosthesis. At the most recent radiological follow-up, 27 shoulders had no glenoid lucencies, and nine had a variety of glenoid lucent lines. In these nine cases, six were excellent, two were satisfactory, and one was unsatisfactory. In five cases these lucent lines were $1 \mathrm{~mm}$ wide and incomplete, in one case it was $1 \mathrm{~mm}$ wide and complete, and in three cases it was $1.5 \mathrm{~mm}$ wide and incomplete. When post-operative pain and movement were compared, no differences were found between the patients with glenoid lucent lines and those without them (pain $p=0.58$, elevation $p=0.64$, external rotation $\mathrm{p}=0.63$, internal rotation $\mathrm{p}=0.23$ ). Four other shoulders had a radiologically loosened glenoid component with a complete lucent line $1.5 \mathrm{~mm}$ or more wide and migration of the glenoid component. One of these patients also had an avulsion fracture of the greater tuberosity. Two of these last patients rated their postoperative pain as severe, one as moderate and the fourth as slight. These four patients declined further surgery. There was also one case of subsidence of the humeral component without clinical consequences. Two patients had marked superior subluxation of the humeral head. Both had an excellent result and no further treatment was deemed necessary.

At the time of completing this study, 39 patients with 40 shoulder replacements had died from causes unrelated to the operation at a mean of 7.5 years after surgery $(0.8$ to 16.4 years). Of these, 32 shoulders had experienced no significant clinical complications and the replacement lasted for the duration of their life. Of the remaining 11 surviving shoulders, at a mean follow-up of 7.3 years, none have had significant clinical complications. The five- ten- and 15 -year patient survival with $95 \%$ CI was $75 \%(63 \%$ to $87 \%)$ at five years, $35 \%(21 \%$ to $49 \%)$ at ten years and $15 \%(1 \%$ to $29 \%)$ at 15 years.

Of the three patients who underwent revision, two of whom required more than one additional procedure, this was undertaken at six months, nine months and four years after the primary arthroplasty.

\section{Discussion}

Total shoulder replacement in these elderly patients proved to be relatively safe, with no peri-operative deaths despite the high prevalence of comorbidities. The hospital stay was prolonged, especially in patients operated on before 1995 . At our institution patients are currently in hospital for between one and three days after primary TSR unless medical complications occur. A stay in the intensive-care unit was needed for five of the 44 patients. The average preoperative haemoglobin value was relatively low at $13.2 \mathrm{~g} / \mathrm{dl}$, (normal values in our laboratory are $13.5 \mathrm{~g} / \mathrm{dl}$ to $17.5 \mathrm{~g} / \mathrm{dl}$ in men and $12.0 \mathrm{~g} / \mathrm{dl}$ to $15.5 \mathrm{~g} / \mathrm{dl}$ in women), with a number of patients requiring post-operative blood transfusion, ${ }^{14}$ seemingly related to this lower pre-operative haemoglobin level. Advanced age has been recently described as an independent risk factor for blood transfusion. ${ }^{15}$ Glenoid resurfacing could be related also to the increase of blood loss in our sample, as has been previously described. ${ }^{16}$ In one study ${ }^{2}$ patients over 80 undergoing conservative humeral resurfacing alone obtained good results, with fewer requirements for blood transfusion and no major medical complications. Preparation of the humeral canal could also have some impact on blood loss compared to the above-mentioned study. Medical complications occurred in nine patients, 
including one myocardial infarction. ${ }^{17}$ The blood loss was a possible contributor to the cardiac problems. No complications were caused by the transfusions. The greater improvement in function and pain found with TSR compared with hemi-arthroplasty in the general population ${ }^{18,19}$ has not been studied in this specific age group.

The morbidity in our elderly group was low, with no peri-operative deaths, in line with a study describing a 1:1000 mortality rate within 90 days of shoulder arthroplasty ${ }^{20}$ when patients with malignancy were excluded. However, there are no studies in patients over the age of 80 with arthritis comparing TSR with stemmed hemiarthroplasty in terms of morbidity and function. We can only speculate that the functional result of TSR may be better than those of stemmed hemi-arthroplasty, with an acceptable morbidity in this age group.

There was a relatively high frequency of glenoid erosion, with nine of these shoulders having augmentation with a bone graft at operation. Cement fixation of the humeral components, although more frequent than in our current practice, was not often required. There were four cases with small- to medium-sized rotator cuff tears. This is in agreement with the prevalence of this problem reported in the literature for the general population undergoing shoulder arthroplasty for OA. ${ }^{21}$

Radiologically, in four shoulders there was loosening and migration of the glenoid component. These were cemented components, two being metal backed and two all-poly designs. Two had pre-operative posterior bony deficiency, with both having impaction cancellous autografting at the time of surgery. The other two had no pre-operative glenoid deficiency. Interestingly, none of the six shoulders with corticocancellous grafts had glenoid loosening, and two of the three shoulders with cancellous impaction grafts developed this complication $(\mathrm{p}=0.08)$. Two of eight shoulders with posterior glenoid deficiency developed glenoid loosening, compared with two of 37 shoulders without significant glenoid erosion. Posterior glenoid deficiency did not reach statistical significance when its association with glenoid loosening was tested $(p=0.11)$, but a tendency should be noted in this regard. An additional ten shoulders had lucent lines around a portion of the glenoid component. ${ }^{22}$ Only two of these had pre-operative glenoid deficiency, and one was the other shoulder in which cancellous graft was used. Nine of these ten had cemented components, five all-polyethylene, and five metal backed. Taking into consideration the sample size of this study, these findings do raise some concern that pre-operative erosion of the glenoid and the technique of grafting the glenoid may be associated with loosening of the component and a poor outcome in this age group.

Post-operative instability occurred in seven shoulders, superior in two and anterosuperior in five. This was clinically significant in four of those with anterosuperior instability, and two required revision. This relatively high frequency of instability may be related to deficiency of the rotator cuff, the inability of these elderly patients to comply with post-operative protection and lack of active use during the healing period. Those patients requiring revision for instability could have been treated with reverse implants to compensate for insufficiency of the rotator cuff, ${ }^{23}$ if the glenoid bone stock had remained adequate.

Overall, TSR for primary OA in these elderly patients was effective in reducing pain and improving movement. The peri-operative period was fraught with additional needs for health care and complications. Fortunately, death was avoided in all patients. There was a higher frequency of erosion of glenoid bone and difficulty with fixation of the glenoid component. There was more post-operative instability than expected. Even in the face of these findings the need for revision surgery was low, with most patients enjoying a comfortable and more functional shoulder until the time of their death for unrelated reasons.

No benefits in any form have been received or will be received from a commercial party related directly or indirectly to the subject of this article.

\section{References}

1. Cofield RH, Hattrup SJ, Sanchez-Sotelo J, Steinmann SP. Shoulder arthroplasty for arthritis. In: Morrey BF, ed. Joint replacement arthroplasty. Vol. 1, Third ed. Rochester: Churchill Livingstone, 2003:438-49.

2. Mullett H, Levy 0, Raj D, et al. Copeland surface replacement of the shoulder: results of an hydroxyapatite-coated cementless implant in patients over 80 years of age. J Bone Joint Surg [Br] 2007;89-B:1466-9.

3. Churchill RS. Elective shoulder arthroplasty in patients older than ninety years of age. J Shoulder Elbow Surg 2008;17:376-9.

4. Sperling JW, Cofield RH, Rowland CM. Neer hemiarthroplasty and Neer total shoulder arthroplasty in patients fifty years old or less: long-term results. J Bone Joint Surg $[A m]$ 1998;80-A:464-73.

5. Raiss P, Aldinger PR, Kasten P, Rickert $\mathbf{M}$, Loew $\mathbf{M}$. Total shoulder replacement in young and middle-aged patients with glenohumeral osteoarthritis. J Bone Joint Surg [Br] 2008;90-B:764-9.

6. Deshmukh AV, Koris M, Zurakowski D, Thornhill TS. Total shoulder arthroplasty: long-term survivorship, functional outcome, and quality of life. J Shoulder Elbow Surg 2005;14:471-9.

7. Haines JF, Trail IA, Nuttall D, Birch A, Barrow A. The results of arthroplasty in osteoarthritis of the shoulder. J Bone Joint Surg [Br] 2006;88-B:496-501.

8. Norris TR, lannotti JP. Functional outcome after shoulder arthroplasty for primary osteoarthritis: a multicenter study. J Shoulder Elbow Surg 2002;11:130-5.

9. Yamada M, Briot J, Pedrono A, et al. Age- and gender-related distribution of bone tissue of osteoporotic humeral head using computed tomography. J Shoulder Elbow Surg 2007; 16:596-602

10. Sperling JW, Cofield RH, Schleck CD, Harmsen WS. Total shoulder arthroplasty versus hemiarthroplasty for rheumatoid arthritis of the shoulder: results of 303 consecutive cases. J Shoulder Elbow Surg 2007;16:683-90.

11. Sperling JW, Cofield RH, O'Driscoll SW, Torchia ME, Rowland CM. Radiographic assessment of ingrowth total shoulder arthroplasty. J Shoulder Elbow Surg 2000;9:507-13.

12. Keats AS. The ASA classification of physical status: a recapitulation. Anesthesiology 1978;49:233-6.

13. Gill DR, Cofield RH, Rowland C. The anteromedial approach for shoulder arthroplasty: the importance of the anterior deltoid. J Shoulder Elbow Surg 2004;13:532-7.

14. Sperling JW, Duncan SF, Cofield RH, Schleck CD, Harmsen WS. Incidence and risk factors for blood transfusion in shoulder arthroplasty. J Shoulder Elbow Surg 2005;14:599-601.

15. Gruson KI, Accousti KJ, Parsons BO, Pillai G, Flatow EL. Transfusion after shoulder arthroplasty: an analysis of rates and risk factors. J Shoulder Elbow Surg 2009;18:225-30.

16. Gartsman GM, Roddey TS, Hammerman SM. Shoulder arthroplasty with or without resurfacing of the glenoid in patients who have osteoarthritis. J Bone Joint Surg [Am]2000;82-A:26-34.

17. Sperling JW, Cofield RH. Pulmonary embolism following shoulder arthroplasty. J Bone Joint Surg [Am] 2002;84-A:1939-41. 
18. Bryant $D$, Litchfield $R$, Sandow $M$, et al. A comparison of pain, strength, range of motion, and functional outcomes after hemiarthroplasty and total shoulder arthroplasty in patients with osteoarthritis of the shoulder: a systematic review and metaanalysis. J Bone Joint Surg [Am] 2005;87-A:1947-56.

19. Edwards TB, Kadakia NR, Boulahia A, et al. A comparison of hemiarthroplasty and total shoulder arthroplasty in the treatment of primary glenohumeral osteoarthritis: results of a multicenter study. J Shoulder Elbow Surg 2003;12:207-13.

20. White CB, Sperling JW, Cofield RH, Rowland CM. Ninety-day mortality after shoulder arthroplasty. J Arthroplasty 2003;18:886-8.
21. Edwards TB, Boulahia A, Kempf JF, et al. The influence of rotator cuff disease on the results of shoulder arthroplasty for primary osteoarthritis: results of a multicenter study. J Bone Joint Surg [Am] 2002;84-A:2240-8.

22. Mileti J, Boardman ND 3rd, Sperling JW, et al. Radiographic analysis of polyethylene glenoid components using modern cementing techniques. J Shoulder Elbow Surg 2004;13:492-8.

23. Levy JC, Virani N, Pupello D, Frankle M. Use of the reverse shoulder prosthesis for the treatment of failed hemiarthroplasty in patients with glenohumeral arthritis and rotator cuff deficiency. J Bone Joint Surg [Br] 2007;89-B:189-95. 